\title{
Stevens-Johnson syndrome/toxic epidermal necrolysis presumably induced by norfloxacin
}

\author{
Jolanta Maciejewskaํ, Marek Jankowski¹, Barbara Zegarska², Rafał Czajkowski ${ }^{1}$
}

${ }^{1}$ Department of Dermatology, Sexually Transmitted Diseases and Immunodermatology, Ludwik Rydygier Medical College in Bydgoszcz, Nicolaus Copernicus University in Torun, Poland Head of Department: Rafał Czajkowski MD, PhD, DSc, Assoc. Prof.

${ }^{2}$ Department of Cosmetology and Esthetic Dermatology, Ludwik Rydygier Medical College in Bydgoszcz, Nicolaus Copernicus University in Torun, Poland

Head of Department: Barbara Zegarska MD, PhD, Prof. NCU

Postep Derm Alergol 2014; XXXI, 3: 194-196

DOI: $10.5114 /$ pdia.2014.40796

\begin{abstract}
Stevens-Johnson syndrome (SJS) and toxic epidermal necrolysis (TEN) are rare mucocutaneous, unpredictable, life-threatening drug side reactions. A very rare case of norfloxacin-induced SJS/TEN overlap with uncommon clinical presentation in a patient with a suspected urinary tract infection is reported. The SJS/TEN management with possible treatment capabilities are discussed. In spite of only two previous reports of norfloxacin-induced SJS and TEN we believe that awareness of norfloxacin adverse reactions needs to be raised among prescribers.
\end{abstract}

Key words: Stevens-Johnson syndrome, toxic epidermal necrolysis, norfloxacin.

\section{Introduction}

Stevens-Johnson syndrome (SJS) and toxic epidermal necrolysis (TEN) are rare but potentially lethal acute adverse drug reactions of differing severity, characterized by erythema and epidermal detachment of the skin and mucous membranes. The SIS and TEN are considered the same disease process; the distinction is made based on body surface area involvement. The SJS is characterized by $<10 \%$ of the body surface area of epidermal detachment, SJS/TEN overlap by 10-30\%, and TEN by $>30 \%$ [1]. The most commonly implicated drugs are carbamazepine, allopurinol, co-trimoxazole, lamotrigine, nevirapine, sulfonamides, cephalosporins and tetracyclines. To our knowledge, this is the third case of SJS/TEN spectrum that could be related to the use of oral norfloxacin [2-4].

\section{Case report}

A 68-year-old man presented with multiple non-coalescing macular lesions on the skin of the trunk, face and upper extremities of 2 days' duration (Figure 1). Based on anamnesis he was taking atorvastatin (used regularly for the last year) and Neurovit (thiamine hydrochloride, pyridoxine hydrochloride, cyanocobalamin) (used periodically for the last few months, last doses taken in the preceding week), and for 4 days before the onset of lesions $400 \mathrm{mg}$ norfloxacin twice daily, because of a suspected urinary tract infection.

On admission, lesions covered $20 \%$ of the body surface area. One day later, lesions extended over the scrotum, perianal area and palmar area. Flaccid blisters and erosions developed on the base of erythematous lesions (Figure 2). The Nikolsky's sign was positive. Within the next 2 days, conjunctival hyperemia and blisters, erosions and ulcerations of the oral mucosa appeared leading to difficulties in swallowing and visual impairment. The SCROTEN scale score was 3 points and 2 points on admission and third day of hospitalization, respectively. General physical examinations were normal, except heart rate $(130 / \mathrm{min})$.

Laboratory findings upon admission revealed leucocytosis, hypoalbuminemia and hypokalemia. Histopathological examination revealed necrotic keratinocytes, vacuolar degeneration in the basal layer, papillary dermal edema and lymphocytic perivascular infiltrates. Civatte bodies in epidermis were present confirming keratinocyte apoptosis (Figure 3). Indirect immunofluorescence test was negative. Direct immunofluorescence showed

Address for correspondence: Rafał Czajkowski MD, PhD, DSc, Assoc. Prof., Department of Dermatology, Sexually Transmitted Diseases and Immunodermatology, Ludwik Rydygier Medical College in Bydgoszcz, Nicolaus Copernicus University in Torun, 9 Skłodowskiej-Curie St, 85-094 Bydgoszcz, Poland, phone: +48 5258545 68, e-mail: r.czajkowski@cm.umk.pl Received: 17.10.2013, accepted: 15.12.2013. 




Figure 1. Left side of the face with macular and exfoliative skin lesions

no immune complexes neither in epidermis nor in the dermal-epidermal border. Blood, throat and urine cultures were also negative. Based on anamnesis, clinical appearance and histopathological image analysis, SJS/TEN was diagnosed.

Intravenous methylprednisolone 500 mg daily (2 days) and 250 mg daily (next 3 days), oral cyclosporine A (3 mg/ kg b.w. with serum-level monitoring, 19 days), intravenous fluids and supportive care together with topical therapy for the oral, ocular, and genital mucosa were initiated. Due to massive oral mucosa erosions, parenteral nutrition was also started. The patient was discharged after 30-day hospitalization in overall good condition.

\section{Discussion}

The SIS and TEN are rare mucocutaneous, unpredictable, life-threatening drug side reactions. Uncommonly, the disease is caused by Mycoplasma spp., Herpes simplex virus or Hepatitis $C$ and $B$ viruses infections. The highest mortality among causing drugs was found from antibiotic treatment, the lowest from using non-steroidal anti-inflammatory drugs [5]. Most widespread antibiotics causing SJS/TEN are aminopenicillins, cephalosporins, macrolides, tetracyclines and quinolones (mainly ciprofloxacin) $[6,7]$.

It is very difficult to indicate a drug responsible for SJS/TEN development in the patient taking multiple medications at the same time, as in described case. In most incidents of drug-induced SJS/TEN, initial skin symptoms appear 2-3 weeks after suspicious medication application, but antibiotics can act more rapidly (a few days,

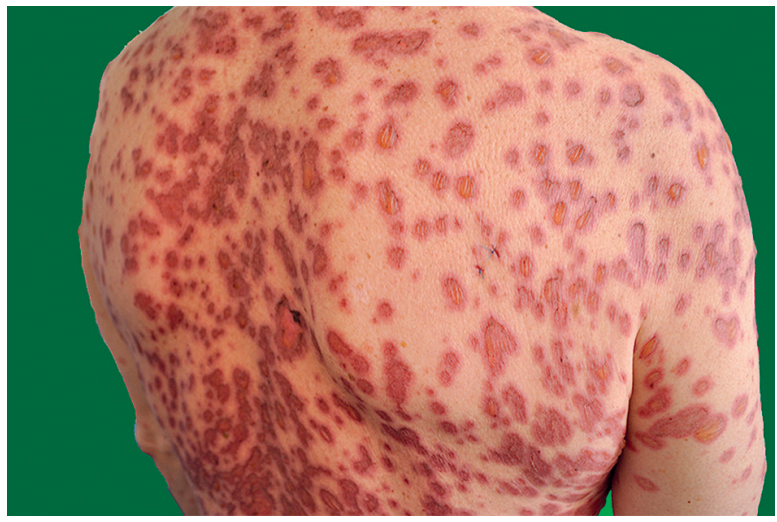

Figure 2. Well-marginated, non-coalescing flaccid blisters and erosions of the trunk developed on the erythematous base. This is a rather uncommon presentation of SJS, more expected in pemphigus

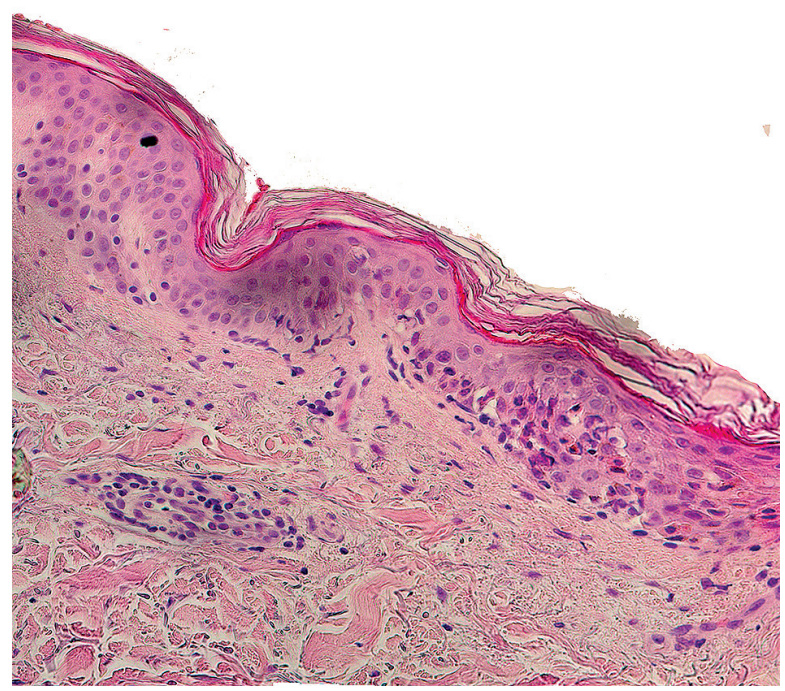

Figure 3. Histopathological examination of a skin sample taken from the right shoulder area revealed necrotic keratinocytes, vacuolar degeneration in the basal layer, papillary dermal edema and lymphocytic perivascular infiltrates. Keratinocyte necrosis is of rather lower intensity than expected in SJS

even after a single dose) [5, 8]. Therefore, we suggest norfloxacin is a causative agent of SJS/TEN in the reported case. No data can be found linking B-group vitamins with SIS or TEN, but probably vitamin B-complex can aggravate the carbamazepine-induced TEN [9]. Our patient was taking B-group vitamins before norfloxacin application, what could intensify epidermal toxic reaction. The mechanism of such possible interaction is not known, and it needs to be investigated in detail.

Several therapies have been proposed for TEN, with contradictory results. Currently, intravenous immunoglob- 
ulins (IVIG) and plasmapheresis are considered most effective $[10,11]$. There is a risk of serious adverse effects with plasmapheresis similar to blood transfusion. Use of corticosteroids is generally controversial, however some authors suggest that corticosteroids are effective if administered within $72 \mathrm{~h}$ from the onset of first symptoms [12].

Cyclosporine $A$ has been used in the treatment of TEN since 1986. It acts by T cells activity decreasing and weakness of immune response as a result of calcineurin inhibition. In a report by Arevalo et al., there was a 0\% mortality rate in TEN patients treated with $3 \mathrm{mg} / \mathrm{kg}$ b.w. of cyclosporine A compared to $50 \%$ mortality in patients treated with methylprednisolone with cyclophosphamide [13]. The use of cyclosporine A was associated with lower levels of hepatic, renal and cardiac injury. We believe that cyclosporine A with short-term early methylprednisolone administration can be successfully used when IVIG and plasmapheresis are contraindicated.

\section{Conclusions}

The SIS and TEN reactions have been reported as adverse effects of several fluoroquinolones. Despite no hard evidence of drug lymphocyte stimulation test results and only two previous reports of norfloxacin-induced SJS and TEN we believe that awareness of norfloxacin adverse reactions needs to be raised among prescribers [2, 3]. Norfloxacin-induced SJS may mimic pemphigus, what makes diagnosis more challenging.

\section{Conflict of interest}

There is no conflict of interest.

\section{References}

1. Roujeau JC. Stevens-Johnson syndrome and toxic epidermal necrolysis are severity variants of the same disease which differs from erytema multiforme. I Dermatol 1997; 24: 726-9.

2. Kubo-Shimsaki A, Yoshimoto K, Tatsumi E, et al. Norfloxacin-induced infectious mononucleosis (IM)-like syndrome with Stevens-Johnson syndrome. Rinsho-Ketsueki 1992; 33: 823-8.

3. Sahin MF, Ozturkcan S, Inanir I, et al. Norfloxacin-induced toxic epidermal necrolysis. Ann Pharmacother 2005; 39: 768-70.

4. Czajkowski R, Weiss-Rostkowska V, Wankiewicz A, et al. Stevens-Johnson syndrome induced by carbamazepine. Acta Pol Pharm 2007; 64: 89-92.

5. Diaz L, Ciurea AM. Cutaneous and systemic adverse reactions to antibiotics. Dermatol Ther 2012; 25: 12-22.

6. Harr T, French LE. Toxic epidermal necrolysis and Stevens-Johnson syndrome. Orphanet J Rare Dis 2010; 5: 39.

7. Win A, Evers ML, Chmel H. Stevens-Johnson syndrome presumably induced by ciprofloxacin. Int I Dermatol 1994; 33 : 512-4.

8. Moshfeghi M, Mandler HD. Ciprofloxacin-induced toxic epidermal necrolysis. Ann Pharmacother 1993; 27: 1467-9.

9. Fegade MV, Bhounsule SA, Pereira I. Can vitamin B-complex aggravate the carbamazepine induced toxic epidermal necrolysis? J Clin Diagn Res 2012; 6: 1571-3.
10. Fromowitz JS, Ramos-Caro FA, Flowers FP. Practical guidelines for the management of toxic epidermal necrolysis and Stevens-Johnson syndrome. Int I Dermatol 2007; 46: 1092-4.

11. Knowles S, Shear NH. Clinical risk management of Stevens-Johnson syndrome/toxic epidermal necrolysis spectrum. Dermatol Ther 2009; 22: 441-51.

12. Schwartz RA, McDonough PH, Lee BW. Toxic epidermal necrolysis: Part II. Prognosis, sequelae, diagnosis, differential diagnosis, prevention, and treatment. J Am Acad Dermatol 2013; 69: 187.e1-6.

13. Arevalo JM, Lorente JA, Gonzales-Herrada C. Treatment of toxic epidermal necrolysis with cyclosporin A. J Trauma 2000; 48: 473-8. 